\title{
Intervenções para o Fenômeno Bullying na Infância: Uma Revisão Sistemática da Literatura
}

\author{
Juliana da Rosa Pureza 1 \\ Universidade do Vale do Rio dos Sinos \\ Angela Helena Marin ${ }^{2}$ \\ Universidade do Vale do Rio dos Sinos \\ Carolina Saraiva de Macedo Lisboa ${ }^{3}$ \\ Pontifícia Universidade Católica do Rio Grande do Sul
}

\begin{abstract}
RESUMO
O bullying está associado a consequências negativas para o desenvolvimento infantil. Encontra-se na literatura estudos recentes propondo intervenções para o manejo do bullying. Este estudo teve como objetivo apresentar uma revisão sistemática da literatura de 2002 a 2012 sobre intervenções para o bullying na infância, que resultou em 19 artigos científicos. Dois juízes analisaram o objetivo, a abordagem teórica, a temática e os aspectos metodológicos das intervenções. A maioria objetivou a diminuição dos índices de bullying, ocorreu na escola, em grupo, envolvendo toda a escola. Constatou-se diversidade de instrumentos de avaliação, e dificuldades em identificar modelos teóricos das intervenções, além de nenhum estudo realizado com crianças pré-escolares. São sugeridas possibilidades de pesquisas futuras acerca de intervenções para o bullying.
\end{abstract}

Palavras-chave: bullying, intervenção, infância.

\begin{abstract}
Interventions to bullying phenomenon in childhood: a systematic review of literature

Bullying is associated with negative consequences for psychosocial development of children. There are recent studies in literature with proposals for management of bullying. This study aims to present a systematic review of the literature between years 2002 to 2013 about interventions for bullying, and final selection included 19 scientific articles. Two judges analyzed objective, theoretical approach, thematic and methodological aspects of interventions. Was observed that most interventions aimed to reduce rates of bullying, occurred at school, in group, and involve whole school. There is a wide range of assessment instrument, and difficult to identify theoretical models of interventions, and no study has been conducted with pre-scholar children. Possibilities are suggested for future research about bullying interventions on childhood.
\end{abstract}

Keywords: bullying, intervention, childhood.

O conceito de bullying compreende atos violentos, físicos ou psicológicos, praticados entre pares, em que o agressor agride sistematicamente a mesma vítima com a intenção de ferir, machucar ou magoar sem motivação aparente; como consequência, essa violência acaba gerando discriminação e exclusão da criança por parte do grupo (Olweus, 1993). Essas agressões ocorrem com desigualdade de poder, onde os agressores estão em maior número ou são mais fortes física e/ou psicologicamente, deixando a vítima subjugada e sem defender-se (Craig \& Harel, 2004; Olweus, 1993).

É importante salientar que o bullying é fenômeno social e que suas definições são influenciadas por aspectos culturais que variam conforme o ambiente e o contexto social (Binsfeld \& Lisboa, 2010; Lopes, 2005). No Japão, por exemplo, o bullying é definido como uma posição dominante de poder, enquanto na França inclui todas as possíveis incivilidades que permeiam o ambiente escolar (Catini, 2004). No Brasil, observa-se que as crianças agressoras tendem a ser mais deprimidos do que as vítimas, dado que se difere da literatura internacional, sugerindo especificidades culturais como, por exemplo, a negligencia por parte da comunidade escolar (Binsfeld \& Lisboa, 2010). 
Atualmente, os estudos de prevalência indicam uma alta frequência do bullying nas escolas. Os resultados do Relatório Internacional da Saúde Mundial (Craig \& Harel, 2004) apontam o bullying como um problema mundial que afeta aproximadamente um terço das crianças. Em estudos brasileiros, essa porcentagem apresenta uma variação de um terço de crianças que já se envolveram em alguma situação de bullying (Francisco \& Libório, 2009, Kristensen, et al. 2009, Malta et al., 2010). Craig e Harel (2004) ressaltam ainda que, mesmo que esses índices de prevalência estejam se mantendo estáveis, a frequência de ocorrências de bullying tem aumentado. Outros estudos demonstram que, no Brasil, os estudos sobre bullying são recentes, motivo pelo qual a maioria dos brasileiros desconhece o tema e sua gravidade (Binsfeld \& Lisboa, 2010, Kristensen, et al. 2009, Lisboa, 2005, Lisboa, Braga \& Ebert, 2009, Malta et al., 2010).

Por ser uma agressão entre pares, o bullying ocorre frequentemente em escolas ou ambientes que favoreçam as relações em grupo (Rolim, 2008). Salmivalli, Lagerspetz, Bjorkqvist, Osterman e Kaukiainen (1996) classificam os participantes envolvidos no bullying em diferentes papeis, a saber: o agressor, a vítima e a vítima-agressora (sofre e pratica algum tipo de bullying), além do incentivador (toma a iniciativa), dos seguidores (unem-se aos agressores), dos reforçadores (encorajam o agressor), dos defensores (ajudam a vítima) e dos espectadores (assistem sem reagir).

Em função de se caracterizar como uma prática de violência que gera importantes implicações físicas e emocionais para os envolvidos, diversos estudos vêm sendo realizados com o objetivo de identificar as consequências do bullying, destacando-se a depressão e a baixa autoestima (Binsfeld \& Lisboa, 2010), além de problemas na vida adulta associados a comportamentos antissociais, instabilidade no trabalho, relacionamentos afetivos pouco duradouros e consequências legais (Lopes, 2005). Além disso, quando o bullying ocorre na infância, ele pode agravar problemas já existentes ou desencadear transtornos psicológicos e dificuldades de aprendizagem (Binsfeld \& Lisboa, 2010; Caballo, Calderero, Carrillo, Salazar, \& Irurtia, 2011).

A alta prevalência do bullying tem sido associada a crianças, de até 12 anos, principalmente entre os matriculados em escolas de ensino fundamental, apresentando queda com o seu crescimento e amadurecimento (Craig \& Harel, 2004; Cross et al.,
2011; Francisco \& Libório, 2009; Kristensen, Schaefer, Rigoli, Busnello, \& Calbo, 2009; Malta et al., 2010; Rigby, 1997; Whitney \& Smith, 1993). A maior incidência na infância pode ser entendida devido à mudança de interesses e um domínio de atividades extraescolares ao longo do desenvolvimento, com o aumento da idade (Craig \& Harel, 2004; Lisboa, Braga, \& Ebert, 2009; Malta et al., 2010; Rolim, 2008; Olweus, 1993).

Nesse sentido, encontra-se na literatura estudos recentes apresentando propostas de intervenções para o manejo e o tratamento do bullying com participantes da referida faixa etária (Caballo et al., 2011; Joronen, Konu, Rankin, \& Stedt-Kurki, 2012; Kärnä et al., 2011a; Kärnä et al., 2011b; Ttofi \& Farrington, 2011). Contudo, apesar da relevância do assunto e do crescente interesse científico pelo tema, os resultados dessas intervenções ainda são modestos, não havendo consenso sobre a melhor estratégia para intervir em relação ao bullying (Merrell, Gueldner, Ross, \& Isava, 2008; Ttofi \& Farrington, 2011). Muitas intervenções documentadas produziram um efeito importante sobre a vitimização, mas esse ainda é considerado pequeno, visto que não parece atingir todos os papéis envolvidos no bullying, como os agressores e as testemunhas (Ttofi \& Farrington, 2011). Frente ao exposto, o presente estudo teve como objetivo realizar uma revisão sistemática da literatura, considerando o período entre os anos de 2002 a 2012 sobre intervenções para o fenômeno bullying na infância.

\section{MÉTODO}

O levantamento bibliográfico realizado para este estudo contemplou as seguintes bases de dados: MEDLINE, LILACS, SCIELO, ASP (EBSCO), Oxford University Press, PsycINFO, ScienceDirect e Web of Science. A busca foi realizada por meio dos seguintes descritores e operadores booleanos: bullying $A N D$ treatment, bullying AND therapy, bullying AND intervention.

Foram incluídos todos os artigos empíricos publicados em periódicos científicos que tivessem como objetivo apresentar ou avaliar alguma intervenção ou tratamento para o fenômeno bullying na infância e que estivessem publicados nas línguas portuguesa, inglesa ou espanhola. Quanto ao período de publicação dos mesmos, considerou-se um intervalo de onze anos, compreendidos entre janeiro de 2002 a 2013. Foram excluídos os artigos cujo tema não contemplasse $o$ 
objetivo proposto neste estudo ou que não enfocassem a população infantil, que aqui é considerada até a idade de 12 anos, conforme critério do ECA (1990). Artigos de revisão teórica ou sistemática, teses, dissertações, livros ou outros documentos também não foram incluídos.

Conforme consta na Figura 1, inicialmente, foram identificados 201 artigos. Destes, 26 foram excluídos por serem duplicatas e cinco por estarem disponíveis em outro idioma que não o português, inglês ou o espanhol. Após a aplicação desta primeira seleção, 170 artigos permaneceram, os quais foram analisados quanto aos seguintes critérios de inclusão, considerando os seus títulos e resumos: bullying ser a temática principal, apresentar ou avaliar uma intervenção/tratamento e ter crianças como população alvo. Após esta segunda seleção, 26 artigos permaneceram, dos quais sete não estavam disponíveis na íntegra online gratuitamente. Desta forma, a seleção final contou com 19 artigos. Todos os estudos selecionados foram analisados de acordo com o objetivo e critérios pré-estabelecidos do presente estudo.

A partir da seleção final, foi realizada a análise temática, em que todos os artigos foram analisados por dois juízes independentes que preencheram uma ficha de leitura após leitura exaustiva do artigo. Os procedimentos desta etapa da análise de dados foram baseados em Triviños (1987), e consistem na préanálise (realização de uma leitura exaustiva de cada
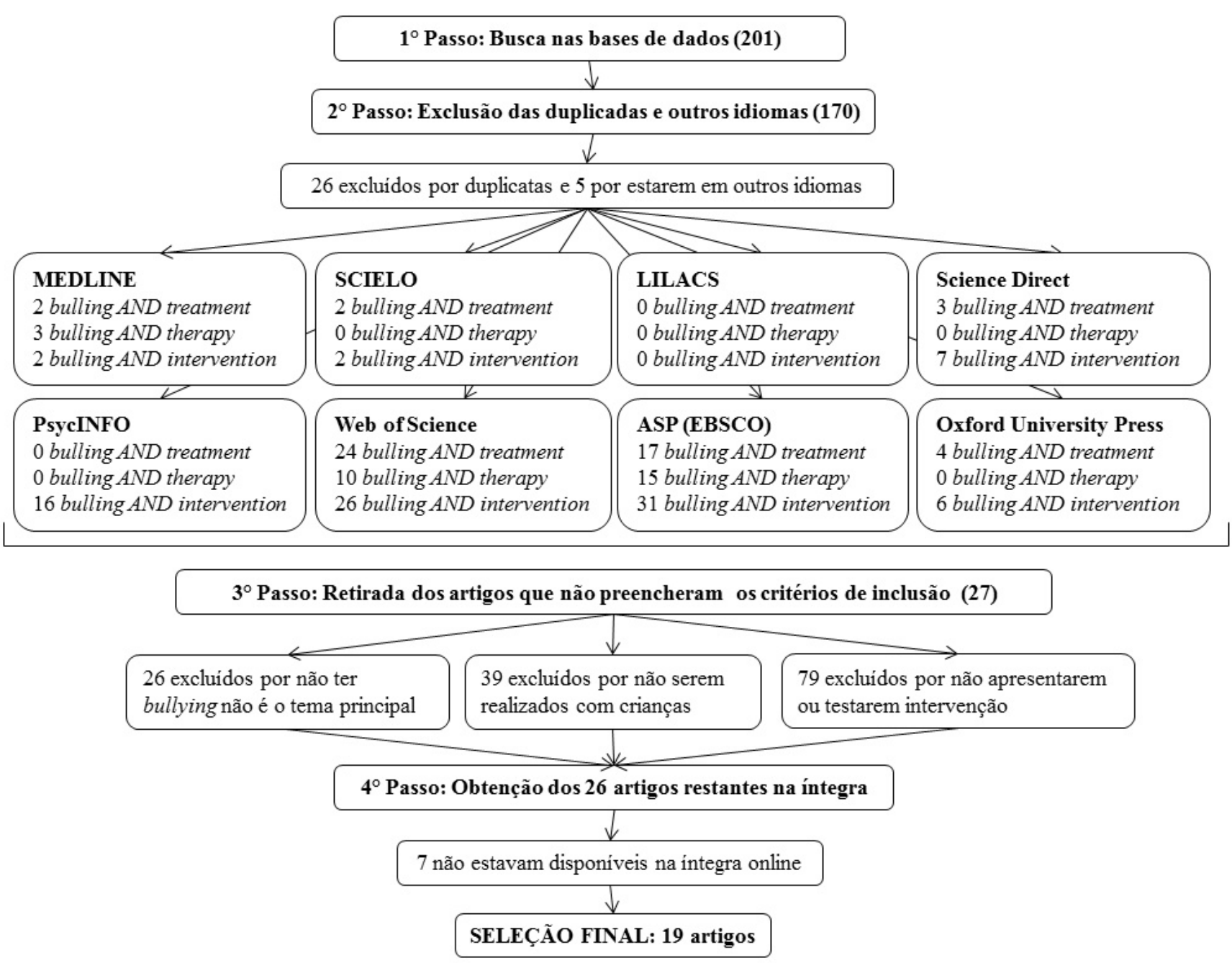

Figura 1. Procedimento de seleção dos artigos revisados. 
artigo e organização dos dados), exploração do material (análise das convergências e divergências entre os estudos) e interpretação teórica dos dados (compreensão teórica e metodológica dos resultados encontrados).

\section{RESULTADOS}

Após a realização da pré-analise, a partir da qual o material identificado foi avaliado, foi realizada a exploração e organização do material, agrupando-o de acordo com as convergências e divergências encontradas (Triviños, 1987). Para esta etapa da análise, foram consideradas as seguintes categorias definidas a priori: objetivo, abordagem teórica, temática e aspectos metodológicos das intervenções (local, participantes, formato e procedimentos utilizados para avaliação do bullying), além da identificação dos fenômenos psicológicos associados ao bulliyng.

Com relação ao objetivo dos estudos identificados nesta revisão, pode-se observar que a maioria (16) deles visava à diminuição dos índices de bullying no contexto escolar (Bauer, Lozano \& Rivara, 2007; Bowllan, 2011; Caballo et al., 2011; Cross et al., 2011; Fung, 2011; Hui, Tsang, \& Law, 2011; Jenson, Dieterich, Brisson, Bender, \& Powell, 2010; Joronen et al., 2012; Kärnä et al., 2011a; Kärnä et al., 2011b; Kim, 2006; McWilliam, 2010; Perkins, Craig, \& Perkins, 2011; Salmivalli, Kärnä, \& Poskiparta, 2011; Tsang, Hui, \& Law, 2011; Waasdorp, Bradshaw, \& Leaf, 2012; Williford et al., 2012). Seis estudos tiveram ainda como objetivo testar intervenções que buscavam desenvolver alguma habilidade específica para o manejo do bullying, como, por exemplo, habilidades sociais, comportamentos antibullying, artes marciais, entre outros (Bauer et al., 2007; Cross et al., 2011; Domino, 2013; Fung, 2011; Kärnä et al., 2011a; McWilliam, 2010), sendo que alguns deles (6) também pretendiam que a intervenção interferisse em aspectos da saúde mental na infância (Domino, 2013; Fung, 2011; Kärnä et al., 2011b; Hui et al., 2011; Joronen et al., 2012; Tsang et al., 2011).

Observou-se que muitas das intervenções analisadas (10) não deixaram explícito qual o modelo teórico que sustentava o desenvolvimento da intervenção (Bauer et al., 2007; Bowllan, 2011; Caballo et al., 2011; Cross et al., 2011; Kärnä et al., 2011a; Kärnä et al., 2011b; Kim, 2006; Perkins et al., 2011; Salmivalli et al., 2011; Williford et al., 2012). Por outro lado, nove intervenções apresentaram o modelo teórico que embasou a intervenção, sendo contemplados o Modelo de Processamento de Informações Sociais (Fung, 2011), o Modelo de Desenvolvimento Social (Jenson et al., 2010), o Modelo do Desenvolvimento Positivo da Juventude (Domino, 2013; Hui et al., 2011; Tsang et al., 2011), o Modelo Sócio-Ecológico (Waasdorp et al., 2012), o Modelo Teórico do Bem-Estar Escolar (Joronen et al., 2012), o Modelo Tradicional das Artes Marciais (Twemlow et al., 2008) e a Jurisprudência Terapêutica (McWilliam, 2010). O Modelo do Desenvolvimento Positivo da Juventude foi o mais contemplado, sendo mencionado por três dos estudos analisados (Domino, 2013; Hui et al., 2011; Tsang et al., 2011).

No que se refere às temáticas abordadas no desenvolvimento das intervenções, foi identificado que metade dos estudos (10) analisados utilizou o treinamento de habilidades sociais (Caballo et al., 2011; Cross et al., 2011; Domino, 2013; Fung, 2011; Hui et al., 2011; Kim, 2006; Jenson et al., 2010; Joronen, et al., 2012; McWilliam, 2010; Tsang et al., 2011). Em contrapartida, aproximadamente um terço (06) optou por usar estratégias de psicoeducação e enfrentamento, tanto para os alunos como para outros profissionais da escola, focando especificamente no bullying (Bauer et al., 2007; Bowllan, 2011; Kärnä et al., 2011a; Kärnä et al., 2011b; Salmivalli et al., 2011; Williford et al., 2012), enquanto a minoria (03) teve como foco outras temáticas distintas, tais como: clima escolar positivo, normas sociais escolares e fundamentos das artes marciais (Perkins et al., 2011; Twemlow et al., 2008; Waasdorp et al., 2012).

Quanto aos aspectos metodológicos das intervenções, destaca-se que todos os estudos foram desenvolvidos no ambiente escolar, mas o papel social do bullying, que foi o foco das intervenções, diferiu. Muitos estudos (09) realizaram a intervenção com toda a escola (professores e alunos), sem diferenciação em relação a quem participava ou não das situações de bullying (Bauer et al., 2007; Cross et al., 2011; Hui et al., 2011; Kärnä et al., 2011a; Kärnä et al., 2011b; McWilliam, 2010; Perkins et al., 2011; Salmivalli et al., 2011; Twemlow et al., 2008). Apenas um estudo envolveu apenas os agressores (Fung, 2011) e outro apenas as vítimas (Kim, 2006). Dois deles trabalharam com a tríade vítima, agressor e testemunha (Bowllan, 2011; Williford et al., 2012) e outros dois somente com a díade vítima e agressor (Jenson et al., 2010; Waasdorp et al., 2012). Também foram identificados quatro estudos que abarcaram toda a turma de alunos na 
intervenção, sem diferenciação com relação ao envolvimento no bullying (Caballo et al., 2011; Domino, 2013; Joronen et al., 2012; Tsang et al., 2011).

No tocante aos participantes das intervenções, constatou-se que um terço das intervenções (06) envolveram pais, professores e outros profissionais da saúde (Caballo et al., 2011; Hui et al., 2011; Kärnä et al., 2011a; Kärnä et al., 2011b; McWilliam, 2010; Williford et al., 2012). Alguns também trabalharam com as crianças, seus pais e professores (Cross et al., 2011; Fung, 2011; Joronen, et al., 2012; Salmivalli et al., 2011), enquanto outros envolveram apenas as crianças (Domino, 2013; Jenson et al., 2010; Kim, 2006; Perkins et al., 2011; Tsang et al., 2011; Twemlow et al., 2008) ou os professores (Bauer et al., 2007; Bowllan, 2011; Waasdorp et al., 2012). Todos os artigos trabalharam com crianças de ambos os sexos, equiparando a amostra do estudo entre meninos e meninas. No que diz respeito à idade, observou-se que a média foi de 11 anos $(\mathrm{DP}=1,49)$, sendo a idade mínima oito anos e a máxima 14 anos.

No que diz respeito ao formato das intervenções analisadas, pode-se observar que todas foram realizadas na modalidade grupal. A maioria (10) dos estudos apresentou formatos pontuais, com um número de encontros compacto que variava de um a 20 encontros, com média de 15,55 encontros ( $\mathrm{DP}=6,28)$ (Caballo et al., 2011; Cross et al., 2011; Domino, 2013; Fung, 2011; Kärnä et al., 2011a; Kärnä et al., 2011b; Kim, 2006; McWillian, 2010; Salmivalli et al., 2011; ; Twemlow et al., 2008; Williford et al., 2012). Já um terço (06) trabalhou com intervenções longas, com duração em torno de um ano (Bauer et al., 2007; Bowllan, 2011; Jenson et al., 2010; Joronen et al., 2012; Perkins et al., 2011; Waasdorp et al., 2012;). Ainda, duas das intervenções não tiveram o período de duração identificado (Hui et al., 2011; Tsang et al. 2011).

A caracterização de cada uma das intervenções está descrita na Tabela 1.

Tabela 1

Caracterização das Intervenções para o Bullying na Infância

\begin{tabular}{llll}
\hline Estudo & País & Duração & Participantes \\
\hline Fung, 2011 & China & $\begin{array}{c}\text { Encontros } \\
\text { pontuais }\end{array}$ & Agressores \\
& & par
\end{tabular}

$\begin{array}{lllll}\text { Jenson et } & \text { Estados } & \text { Encontros } & \text { Vitimas e } & \text { Revised Olweus } \\ \text { al., 2010 } & \text { Unidos } & \text { anuais } & \text { agressores } & \text { BullyNictim } \\ & & & & \text { Questionnaire }\end{array}$

Kärnä et al., Finlândia Encontros Toda a escola $2011^{\mathrm{a}}$ pontuais (20h) avaliação

Reactive and e indicação de professores

Revised Olweus BullyNictim

\section{Instrumentos de Principais resultados}

Proactive Aggression aspectos cognitivos, emocionais e em comportamentos Questionnaire (RPQ) de agressão reativa. Particulares melhorias ocorreram nos componentes de atribuições hostis de outros e graus de sensibilidade a estímulos agressivos. As habilidades de gerenciamento de raiva foram melhoradas.

Não foram encontradas diferenças significativas nas taxas de bullying entre os participantes do grupo controle e experimental. Os comportamentos de bullying diminuíram em ambos os grupos, a queda, no entanto, foi um pouco maior nas escolas que participaram do programa de intervenção. Quanto a redução da vitimização, entre os indivíduos do grupo experimental foi encontrada uma redução significativa, não encontrado no grupo controle.

Comparando as médias do grupo que recebeu a Questionnaire, SelfReported intervenção e o grupo controle, pode-se observar que a mudança ocorreu na vitimização no grupo de intervenção, com uma mudança muito menor no grupo controle.

Victimization, Também houve um aumento da auto-eficácia para a

Provictim Scale, Self- defesa e bem-estar. Verificou-se uma alteração favorável efficacy for defending ao grupo intervenção em todos os outros resultados, scale embora algumas das diferenças foram pequenas Depois de 9 meses de intervenção, que participaram os resultados se mantiveram. 


$\begin{array}{lllll}\begin{array}{l}\text { McWilliam, Austrália } \\ 2010\end{array} & \begin{array}{l}\text { Encontros Toda a escola } \\ \text { anuais }\end{array} & \begin{array}{l}\text { Instrumento não } \\ \text { validado } \\ \text { desenvolvido para o } \\ \text { estudo }\end{array} \\ \text { Kim, 2006 Coreia } & \begin{array}{l}\text { Encontros Vitimas } \\ \text { pontuais } \\ \text { d10) }\end{array} & \begin{array}{l}\text { Peer-Victimization } \\ \text { Scale }\end{array}\end{array}$

Waasdorp Estados Encontros Vitimas e

et al., 2012 Unidos anuais agressores of Classroom
Joronen et Finlândia Encontros Toda a turma Scale of Social al., $2012 \quad$ anuais

Williford et al., 2012

Vitimas, agressores e Victimization e

\section{Finlândia Encontros pontuais} testemunhas

Kärnä et al., Finlândia 2011b

\section{Caballo et} al., 2011

\section{Espanha Encontros} pontuais
Toda a turma

Cuestionario
multimodal de acoso
escolar (CMAE-1)

Não testou os efeitos da intervenção.

Teacher Observation Resultados indicam que as crianças de ambas as

Adaptation Checklist (TOCA-C) condições demonstraram um incremento no risco para envolvimento em bullying e rejeição de pares. Porém, os resultados indicam que as crianças das escolas que receberam o grupo de intervenção apresentaram menos comportamentos de bullying e níveis menores de rejeição durante os quatro anos quando comparadas as crianças das escolas do grupo controle.

Revised Olweus Bully/Nictim Questionnaire

Foram encontrados resultados significativos para estudantes sexo feminino que receberam a intervenção no que diz respeito a prevalência de bullying e exclusão por pares. Não foram encontros resultados para os meninos. Após a intervenção, os professores relataram melhoras para identificar o bullying, e falar com os agressores e vitimas de bullying.

Para o grupo controle, foram encontradas diferenças relationships (SRS) significativas que implicaram em reduções nos papéis de vitimização. Também foi encontrada redução nas variáveis de depressão e ansiedade dos participantes da intervenção. $O$ grupo controle não apresentou os mesmos resultados.

Peer-Reported Houve uma maior diminuição na frequência de bullying no Perception-of-Peers Questionnaire grupo de intervenção no pós-teste em comparação aos resultados do grupo de controle. Tipo de família, classe social, ou educação dos pais não foram associados com o bullying. Depois do programa, a frequência de vitimização diminuiu para o grupo de intervenção.

Os resultados indicam que nas escolas da intervenção diminuíram os percentuais de vitimização e intimidação menores após a intervenção, onde taxas de prevalência de bullying e vitimização foram menores em cada nivel de ensino. Também houve uma queda no índice de assédio moral. Não encontraram-se diferenças entre escolas.

O programa foi eficaz com relação ao bullying, especialmente nos fatores de apoio a vitima, vitimização extrema e testemunha. Em relação ao agressor, 0 programa parece não ter sido eficaz. 


\begin{tabular}{|c|c|c|c|c|c|}
\hline $\begin{array}{l}\text { Tsang et al., } \\
2011\end{array}$ & $\begin{array}{l}\text { Hong } \\
\text { Kong }\end{array}$ & $\begin{array}{l}\text { Não } \\
\text { menciona }\end{array}$ & Toda a escola & Não menciona & Não testou os efeitos da intervenção. \\
\hline $\begin{array}{l}\text { ui et al., } \\
011\end{array}$ & $\begin{array}{l}\text { Hong } \\
\text { Kong }\end{array}$ & $\begin{array}{l}\text { Não } \\
\text { menciona }\end{array}$ & Toda a escola & Não menciona & Não testou os efeitos da intervenção. \\
\hline $\begin{array}{l}\text { erkins et } \\
., 2011\end{array}$ & EUA & $\begin{array}{l}\text { Encontros } \\
\text { anuais }\end{array}$ & Toda a escola & $\begin{array}{l}\text { Instrumento não } \\
\text { validado } \\
\text { desenvolvidos } \\
\text { especificadamente } \\
\text { para o estudo }\end{array}$ & $\begin{array}{l}\text { Das quarto escolas estudadas, apenas uma não } \\
\text { apresentou resultados significativos da mudança da } \\
\text { percepção do bullying. Pode-se observar uma correlação } \\
\text { entre a exposição aos cartazes visuais e no quanto os } \\
\text { índices de bullying diminuíram. }\end{array}$ \\
\hline $\begin{array}{l}\text { almivalli et } \\
., 2011\end{array}$ & Finlândia & $\begin{array}{l}\text { Encontros } \\
\text { pontuais } \\
\text { (20h) }\end{array}$ & Toda a escola & $\begin{array}{l}\text { Revised Olweus } \\
\text { Bully/Nictim } \\
\text { Questionnaire }\end{array}$ & $\begin{array}{l}\text { A porcentagem dos diferentes tipos de bullying diminui no } \\
\text { grupo de intervenção, o que não aconteceu no grupo } \\
\text { controle. Pode-se observar efeito na redução de } \\
\text { cyberbullying. }\end{array}$ \\
\hline $\begin{array}{l}\text { ross et al., } \\
11\end{array}$ & Austrália & $\begin{array}{l}\text { Encontros } \\
\text { anuais }\end{array}$ & Toda a escola & $\begin{array}{l}\text { Instrumento não } \\
\text { validado } \\
\text { desenvolvidos } \\
\text { especificadamente } \\
\text { para o estudo }\end{array}$ & $\begin{array}{l}\text { Os resultados do estudo demonstraram que o grupo de } \\
\text { intervenção, no final do primeiro ano de estudo, estava } \\
\text { menos propenso do que o grupo de comparação para } \\
\text { sofrer bullying, perceber o bullying e denunciar situações } \\
\text { de bullying. O tamanho desses efeitos pode ser } \\
\text { considerado pequeno a moderado. Não foram } \\
\text { encontradas diferenças no final do ano segundo estudo e } \\
\text { no comportamento dos agressores. }\end{array}$ \\
\hline $\begin{array}{l}\text { Twemlow et } \\
\text { al., } 2008\end{array}$ & EUA & $\begin{array}{l}\text { Encontros } \\
\text { pontuais } \\
(03)\end{array}$ & Toda a escola & $\begin{array}{l}\text { Bully-Victim } \\
\text { Questionnaire }\end{array}$ & $\begin{array}{l}\text { Os resultados indicaram que as crianças que participaram } \\
\text { da intervenção apresentaram uma frequência menor de } \\
\text { agressão e testemunha do que as que não participaram. } \\
\text { A participação não foi associada com mudanças na } \\
\text { vitimização. }\end{array}$ \\
\hline $\begin{array}{l}\text { auer, } \\
\text { zano, \& } \\
\text { ivara, }\end{array}$ & EUA & $\begin{array}{l}\text { Encontros } \\
\text { anuais }\end{array}$ & Toda a escola & $\begin{array}{l}\text { Revised Olweus } \\
\text { Bully/Nictim } \\
\text { Questionnaire }\end{array}$ & $\begin{array}{l}\text { Não foram encontrados efeitos após o programa no que } \\
\text { se refere a vitimização, sugerindo que talvez o programa } \\
\text { não esteja funcionando conforme o esperado. }\end{array}$ \\
\hline
\end{tabular}

Ainda foi observado, a partir dos estudos analisados, que existe uma grande diversidade de procedimentos de avaliação e formas de identificar o bullying na infância. Com relação aos instrumentos usados para a avaliação do bullying, o Revised Olweus Bully/Victim Questionnaire (Olweus, 1996) foi o mais utilizado (Bauer et al., 2007; Bowllan, 2011, Fung, 2011; Jenson et al., 2010; Kärnä et al., 2011a; Kärnä et al., 2011b; Salmivalli et al., 2011). Outros 09 instrumentos também foram identificados e aparecem listados na Tabela 1. Todos os estes instrumentos encontrados são de auto-relato (as próprias crianças responderam), tendo sido empiricamente validados anteriormente, apresentando Alpha de Cronbach's que variaram de 0,76 a 0,92 (Bauer et al., 2007; Cabalo et al., 2011; Domino, 2013; Fung, 2011; Kim, 2006;
Joronen, et al., 2012; Twemlow et al., 2008; Waasdorp, Bradshaw, \& Leaf, 2012; Williford et al., 2012).

Além desses, alguns estudos optaram por utilizar questionários ou entrevistas não validados para avaliação do bullying, desenvolvidos especificadamente para o trabalho realizado (Cross et al., 2011; McWilliam, 2010; Perkins et al., 2011). Apenas um dos estudos utilizou a indicação de professores como uma forma de avaliação do bullying (Fung, 2011) e dois não mencionaram como realizaram a sua identificação (Hui et al., 2011; Tsang et al., 2011).

Pode-se observer que alguns estudos (5) avaliaram que as intervenções apresentaram efeitos positivos nos índices globais de bullying (Bowllan, 2011; Perkins et al., 2011; Salmivalli et al., 2011; Waasdorp et al., 2012; 
Williford et al., 2012). No que tange aos papéis sociais, uma grande parte das intervenções (9) apresentou efeito positivo no que diz respeito à vitimização, diminuindo os seus índices (Caballo et al., 2011; Cross et al., 2011; Domino, 2013; Jenson et al., 2010; Joronen et al., 2012; Kärnä et al., 2011a; Kärnä et al., 2011b; Kim, 2006; Williford et al., 2012). No que se refere aos outros papéis no bullying, foram poucos (3) os estudos que apresentaram intervenções que obtiverem efeito nos agressores (Domino, 2013; Fung, 2011; Twemlow et al., 2008), no comportamento de testemunha (Cross et al., 2011; Kärnä et al., 2011b; Twemlow et al., 2008), e no comportamento de intervenção positiva em situações de bullying (Caballo et al., 2011; Cross et al., 2011; Kärnä et al., 2011 $)$. Foi encontrado apenas um estudo que não apresentou nenhum resultado significativo (Bauer, Lozano, \& Rivara, 2007). Ainda, três dos estudos encontrados apenas apresentaram o programa de intervenção, não realizando uma avaliação formal de sua efetividade (Hui et al., 2011; McWilliam, 2010; Tsang et al., 2011).

Ainda, quanto aos outros fenômenos psicológicos pesquisados de forma associada ao bullying nas intervenções, foram identificados estudos que buscaram associá-lo com a depressão (Cross et al., 2011; Williford et al., 2012), as habilidades sociais (Kärnä et al., 2011a; McWilliam, 2010), a ansiedade (Caballo et al., 2011; Cross et al., 2011; Williford et al., 2012), o bem-estar (Hui et al.; 2011; Kärnä et al., 2011a), o autoconceito (Cross et al., 2011; Tsang et al., 2011) e o ajustamento escolar (Cross et al., 2011). No entanto, de um modo geral observa-se que a maioria (11) dos trabalhos revisados optou por não associar o bullying na infância a outros fenômenos psicológicos, pesquisando-o isoladamente (Bauer et al., 2007; Domino, 2013; Fung, 2011; Jenson et al., 2010; Kim, 2006; Joronen, et al., 2012; Kärnä et al., 2011b; Perkins et al., 2011; Salmivalli et al., 2011; Twemlow et al., 2008; Waasdorp et al., 2012).

Foi encontrada uma grande diversidade de países que tem se preocupado em desenvolver e avaliar intervenções para o fenômeno bullying na infância. Identificou-se que a Finlândia (Joronen et al., 2012; Kärnä et al., 2011a; Kärnä et al., 2011b; Salmivalli et al., 2011; Williford et al., 2012) e os Estados Unidos (Bauer et al., 2007; Bowllan, 2011; Domindo, 2013; Jenson et al., 2010; Perkins et al., 2011; Twemlow et al., 2008; Waasdorp et al., 2012) são os países que apresentam maior incidência de intervenções para o fenômeno bullying na literatura. Também foram encontrados estudos provenientes da Austrália (Cross et al., 2011; McWilliam, 2010), China (Fung, 2011; Hui et al., 2011; Tsang et al. 2011), Espanha (Caballo et al., 2011) e Coreia do Sul (Kim, 2006). A distribuição por país das intervenções para o bullying analisadas encontra-se na Figura 2.

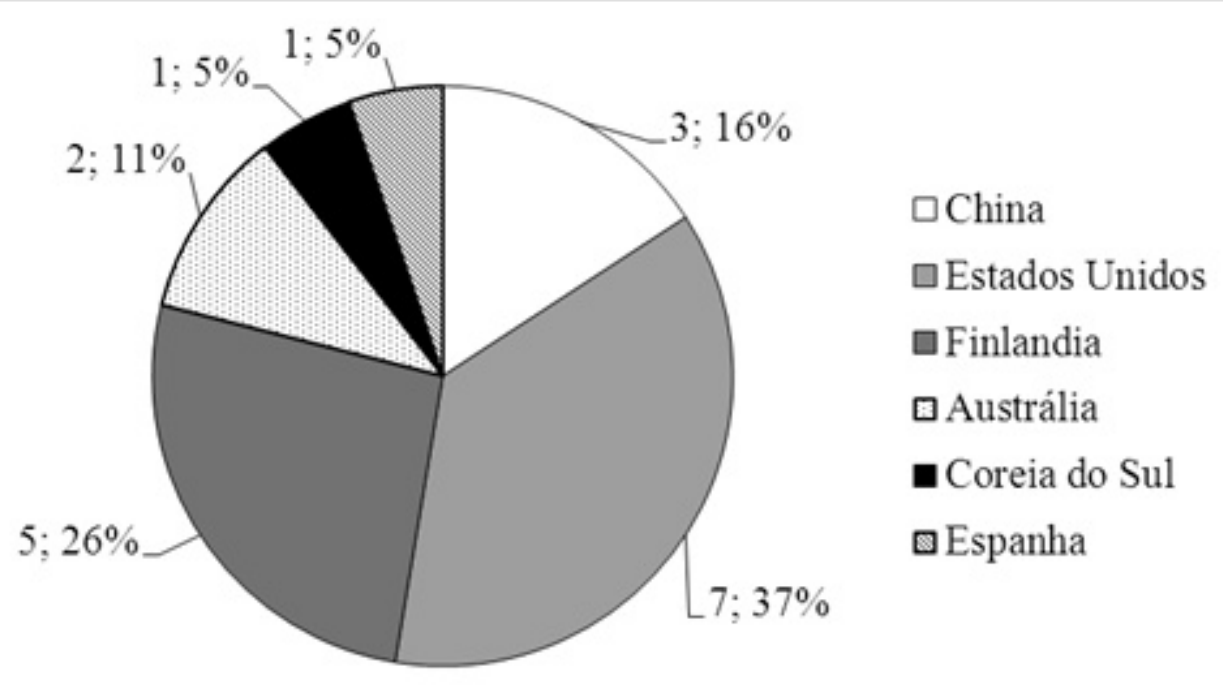

Figura 2. Representatividade das intervenções para o bullying na infância por país. 


\section{DISCUSSÃO}

A partir da revisão sistemática realizada, foi possível observar que a maioria das intervenções para o fenômeno bullying localizadas na literatura datada de 2002 a 2012 teve como objetivo principal a diminuição dos índices de bullying no contexto escolar a partir de intervenções com os participantes diretamente envolvidos. Também se pode identificar que ainda são escassos os estudos que apresentem intervenções para o bullying e que tenham como foco a promoção de saúde na infância, corroborando o modelo clássico dos estudos de psicologia, que privilegia o sofrimento humano. A literatura aponta que a psicologia, em função dos aspectos históricos derivados de sua inserção no mercado de trabalho no período pós-guerra, muitas vezes tende a considerar em suas práticas, principalmente, os aspectos relacionados ao sofrimento humano (Paludo \& Koller, 2007; Seligman \& Csikszentmihalyi, 2000). Contudo, atualmente, pode-se observar o desenvolvimento de intervenções para o tratamento de crianças como uma forma de promoção de saúde mental na vida adulta, diminuindo assim os gastos atuais de controle e assistência em saúde (Del Prette \& Del Prette, 2005; Petersen \& Wainer, 2011).

Nesse sentido, foi possível observar que a abordagem teórica mais utilizada para embasar as intervenções para bullying foi Modelo do Desenvolvimento Positivo da Juventude (Domino, 2013; Hui et al., 2011; Tsang et al., 2011) que, apesar ter sido utilizado por apenas três dos estudos analisados, pode sugerir uma tendência de intervenções para o bullying com foco na promoção de saúde. Porém, de um modo geral não houve uma explicitação clara de quais os modelos fundamentaram $o$ desenvolvimento das intervenções, o que pode dificultar a compreensão dos resultados encontrados.

Já no que se refere às temáticas das intervenções, encontrou-se um predomínio daquelas que trabalharam com as habilidades sociais e com a psicoeducação do bullying. É possível observar que há uma convergência na literatura no que se refere às temáticas trabalhadas nas intervenções para o bullying. Todavia, ainda não existe um consenso sobre os resultados das intervenções robustos para cada um dos papeis envolvidos neste fenômeno (Salmivalli et al., 1996), o que justificaria o desenvolvimento de intervenções fundamentadas em abordagens teóricas específicas, que associassem diferentes temáticas, de modo a formas de manejo mais específicas para os diferentes papéis e possíveis fenômenos psicológicos associados ao bullying.

O bullying é considerado como um fenômeno grupal, que deve ser tratado de forma coletiva (Salmivalli et al., 1996). Em função disso, muitas das propostas analisadas foram realizadas na escola, o que se justifica pelo fato de esse ser um contexto em que o bullying, em geral, emerge e se mantém (Caballo et al., 2011; Salmivalli et al., 1996). Os indivíduos que praticam bullying raramente agem sozinhos, pois essa violência só passa a ser constante e frequente se houver um grupo de apoiadores que a incentiva, encorajando e valorizando o comportamento agressivo. Portanto, o fenômeno bullying deve ser considerado como um problema grupal, emergente de relações que são estabelecidas entre os alunos. Além disso, a opção por trabalhar com todo o grupo escolar abrange não somente as vítimas e os agressores, de modo que essas intervenções possam também reduzir as consequências para as testemunhas e os demais envolvidos, por menor que seja sua participação (Salmivalli et al., 1996). Este dado corrobora a tendência da literatura em direção à adoção de programas universais para prevenir o bullying e atuar no clima escolar, estabelecendo um conjunto de expectativas de comportamento em todos os contextos da escola e envolvendo a todos nas atividades de prevenção (Waasdorp et al., 2012).

Além disso, é possível que outras explicações tenha fomentado esta tendência à adoção de programas de intervenções grupais. A utilização da modalidade grupal também é adotada muitas vezes devido ao fato de o grupo gerar repertórios sociais para os participantes, propiciando os processos de modelagem por meio da observação entre os próprios membros do grupo (Bieling, McCabe \& Antony, 2008; Binsfeld \& Lisboa, 2010; White \& Freeman, 2003). O tratamento grupal muitas vezes é a modalidade que apresenta maior custobenefício, pois um número maior de pacientes pode receber a intervenção com menos profissionais envolvidos e menor infraestrutura (Bieling, McCabe \& Antony, 2008).

No que diz respeito à duração da intervenção, foi constatada uma tendência à intervenções com formatos pontuais, com um número de encontros compacto com média de 15 encontros. Esses resultados corrobora a literatura, que aponta que o número provável de sessões necessárias para atingir mudanças clínicas significativos varia entre 11-18 sessões (Hansen, Lambert \& Forman, 2002). Especialmente em relação às crianças que participaram das intervenções analisadas, foi indicado que a redução da incidência de 
bullying foi maior entre aquelas com idades até 12 anos, sendo a idade de 11 a mais estudada em relação ao fenômeno (Cross et al., 2011). De fato, o bullying é mais prevalente entre alunos com idades entre 11 e 13 anos (Lopes, 2005), o que justificaria o enfoque das intervenções realizadas por esta faixa etária específica. Além disso, estudos apontaram que alunos nessa faixa etária relatam com maior frequência o envolvimento em situações de bullying quando comparados com alunos mais velhos, o que indica um declínio do bullying com o aumento da idade (Cross, et al., 2011; Rigby, 1997; Whitney \& Smith, 1993). Outro aspecto importante é que não foi encontrada nenhuma pesquisa sobre bullying realizada com crianças menores de oito anos. Dados acerca do tema apontam que o bullying é menos frequente na educação infantil e ensino médio (Lopes, 2005), o que corrobora o foco das intervenções no ensino fundamental. Além disso, existe uma dificuldade de acesso e realização de pesquisas com crianças menores em função da não utilização de instrumentos de autorrelato, uma vez que estas ainda estão desenvolvendo a linguagem, dificultando a mensuração dos fenômenos (Kohlsdorf \& Costa Junior, 2009).

Uma das dificuldades metodológicas encontradas nos estudos realizados acerca do fenômeno bullying é a grande diversidade de procedimentos para a sua avaliação e identificação na infância. Observou-se que a maioria dos artigos analisados utilizou o instrumento clássico desenvolvido pelo primeiro pesquisador oficial do tema, Dan Olweus. Identificou-se, também, outros 10 instrumentos para avaliação do bullying mais atuais, o que demonstra um crescente interesse científico na avaliação fidedigna do bullying como uma das formas de verificar a efetividade das intervenções, levando a criação de novas medidas de identificação do fenômeno. É interessante ressaltar que estes instrumentos foram, em sua maioria, validados empiricamente e apresentando uma boa confiabilidade de uso. Porém, esse grande diversidade de instrumentos também permite uma discussão acerca das dificuldades para a replicação e comparação dos resultados de diferentes intervenções, uma vez que as medidas utilizadas são distintas e, muitas vezes, pouco pesquisadas, o que acaba por dificultar o avanço do conhecimento científico na área.

Cabe destacar que a maioria dos estudos analisados não realizou intervenções associando o tratamento do bullying na infância com a avaliação de outros fenômenos psicológicos. As pesquisas sobre bullying relacionam esse fenômeno tanto com sintomas de sofrimento psicológico, como depressão e ansiedade, quanto com fatores de proteção, como habilidades sociais e bem-estar. Entre essas variáveis, a ansiedade foi a mais contemplada, sendo investigada por três estudos com metodologias diversas. Portanto, seriam necessários novos estudos para que se possa compreender o efeito das intervenções em cada variável relacionada ao bullying.

Com relação aos resultados das intervenções, foi possível observar que a maioria dos programas apresentados produziu algum efeito positivo para o bullying. Porém, a maioria destes estudos ainda intervém principalmente no que se refere ao papel da vitima, sendo que os outros papéis no bullying têm sido pouco contemplados, conforme é indicado na literatura (Ttofi \& Farrington, 2011). Da mesma forma, observase que com relação aos papéis as intervenções também divergem nos seus resultados, o que torna difícil a comparação e generalização destes resultados. Dessa forma, pode-se identificar que ainda não existe um consenso sobre a melhor forma de intervir no bullying.

Por fim, é importante salientar que as diferenças culturais relacionadas a cognições, características e comportamentos de crianças e adolescentes envolvidos no bullying são fundamentais para o planejamento de intervenções clínicas eficazes (Binsfeld \& Lisboa, 2010) e que não foram encontradas pesquisas brasileiras propondo intervenções para o bullying na infância. Nesse sentido, sugere-se que sejam realizadas pesquisas nacionais propondo intervenções para o fenômeno bullying adaptadas a realidade brasileira, de modo a conhecer as características que influenciam ou são influenciadas por nossa cultura. É importante que as diferenças culturais sejam levadas em consideração quando comparados e generalizados os dados dos estudos sobre intervenções para o bullying, uma vez que estes se referem a escolas, relações e sociedades distintas.

\section{CONSIDERAÇÕES FINAIS}

O objetivo deste estudo foi realizar uma revisão sistemática a respeito das intervenções para o fenômeno bullying na infância. A partir dos resultados encontrados, acredita-se ser que, para pesquisas futuras, intervenções como foco em papéis específicos para o bullying, com instrumentos de medidas diversificados e empiricamente validados, abrangendo crianças mais novas e com abordagens teóricas bem definidas. Da mesma forma, destaca-se a importância do 
desenvolvimento de pesquisas de intervenção para o bullying dentro do cenário nacional, adaptadas para a realidade brasileira.

Dentre as limitações encontradas neste estudo, podese elencar: o uso de descritores relacionados apenas ao tratamento do fenômeno bullying, o que pode ter excluído do estudo outras intervenções com focos distintos como, por exemplo, a prevenção; a delimitação da faixa etária pelo critério do ECA, o que pode ter feito com que alguns estudos que trabalham com conceitos de infância mais prolongados não tenho sido contemplados; o fato de que esta revisão não avaliou a fundo o nível de impacto e de força de evidência das intervenções encontradas, o que poderia gerar resultados mais completos para um discussão a respeito da efetividade destas intervenções.

Dessa forma, as intervenções publicadas até o momento são encorajadores e estimulantes, o que indica que este é um momento propício para o desenvolvimento de pesquisas com foco em prevenção e promoção de saúde (Ttofi \& Farrington, 2011), em especial no contexto do Brasil.

\section{REFERÊNCIAS}

Allen, K. P. A. (2010). Bullying intervention system: Reducing risk and creating support for aggressive students. Preventing School Failure, 54(3), 199-209.

Bauer, N. S., Lozano, P., \& Rivara, F. P. (2007). The effectiveness of the Olweus Bullying Prevention Program in public middle schools: A controlled trial. Journal of Adolescent Health, 40(3), 266-274.

Binsfeld, A. R., \& Lisboa, C. S. M. (2010). Bullying: Um estudo sobre papéis sociais, ansiedade e depressão no contexto escolar do Sul do Brasil. Interpersona, 4(1), 74105.

Bowllan, N. M. (2011). Implementation and evaluation of a comprehensive, school-wide bullying prevention program in an urban/suburban middle school. Journal of School Health, 81(4), 167-73.

Brasil (1990). Estatuto da Criança e do Adolescente. Lei n ${ }^{\circ}$ 8.069, de 13 de julho de 1990.

Caballo, V. E., Calderero, M., Carrillo, G. B., Salazar, I. C., \& Irurtia, M. J. (2011a). Acoso escolar y ansiedad social en niños (II): Una propuesta de intervención en formato lúdico. Behavioral Psychology, 19(3), 611-626.

Caballo, V. E., Arias, B., Calderero, M., Salazar, I. C., \& Irurtia, M.. J. (2011b). Acoso escolar y ansiedad social en niños (I): Análisis de su relación y desarrollo de nuevos instrumentos de evaluación. Behavioral Psychology, 19(3), 591-609.

Catini, N. (2004) Problematizando o "Bullying" para a realidade brasileira. Tese de Doutorado em Psicologia, Pontifícia Universidade Católica de Campinas, Campinas, SP.
Craig, W. M., \& Harel, Y. (2004). Bullying, physical fighting and victimization. In C. Currie et al. (Eds.), Young people's health in context: International report from the HBSC 2001/02 survey (pp.133-144). WHO Policy Series: Health policy for children and adolescents, Issue 4, Copenhagen: WHO Regional Office for Europe.

Cross, D., Monks, H., Hall, M., Shaw, T., Pintabona, Y., Erceg. E., Hamilton, G., Roberts, C., Waters, S., \& Lester, L. (2011). Three year results of the friendly schools whole\& of \& school intervention on children's bullying behavior. British Educational Research Journal, 37(1), 105-129.

Del Prette, Z. A. P., \& Del Prette, A. (2005a). A importância das habilidades sociais na infância. In Del Prette, Z. A. P., \& Del Prette, A (Eds.), Psicologia das habilidades sociais na infância: Teoria e prática (pp. 270). Petrópolis: Vozes.

Domino, M. (2013) Measuring the Impact of an Alternative Approach to School Bullying. Journal of School Health, 83(6), 430-437.

Francisco, M. V., \& Libório, R. M. C. (2009). Um estudo sobre bullying entre escolares do ensino fundamental. Psicologia: Reflexão \& Crítica, 22(2), 200-207.

Fung, A. L. (2011). Group treatment of reactive aggressors by social workers in a Hong Kong school setting: A twoyear longitudinal study adopting quantitative and qualitative approaches. British Journal of Social Work. 42(8), 1533-1555.

Hui, E. K. P., Tsang, S. K. M., \& Law, B. C. M (2011). Combating school bullying through developmental guidance for positive youth development and promoting harmonious school culture. The Scientific World Journal, $11,2266-2277$.

Jenson, J. M., Dieterich, W. A., Brisson, D, Bender, K. A., \& Powell, A. (2010). Preventing childhood aggression and bullying: implications for school-based intervention research from the Denver public schools trial. Research on Social Work Practice, 20(5), 509-517.

Joronen, K., Konu, A., Rankin, H. S., \& Stedt-kurki, P. (2012). An evaluation of a drama program to enhance social relationships and anti-bullying at elementary school: a controlled study. Health Promotion International, 27(1), 5-14.

Kärnä, A., Voeten, M., Little, T. D., Poskiparta, E., Kaljonen, A., \& Salmivalli, C. (2011a). A large-scale evaluation of the KiVa antibullying program: Grades 4-6. Child Development, 82(1), 311-330.

Kärnä, A., Voeten, M., Little, T. D., Poskiparta, E., Alanen, E., \& Salmivalli, C. (2011b). Going to scale: A nonrandomized nationwide trial of the KiVa antibullying program for grades 1-9. Journal of Consulting and Clinical Psychology, 79(6), 796-805.

Kim, J. U. (2006). The effect of a bullying prevention program on responsibility and victimization of bullied children in Korea. International Journal of Reality Therapy, 26(1), 4-8.

Kristensen, C. H., Schaefer, L. S., Rigoli, M. M., Busnello, F. B., \& Calbo, A. S. (2009). Bullying na escola: Comportamento agressivo, vitimização e conduta prósocial entre pares. Contextos Clínicos, 2(2), 73-80. 
Kohlsdorf, M., \& Costa Junior, A. L. (2009). O autorrelato na pesquisa em psicologia da saúde: Desafios metodológicos. Psicologia Argumento, 27, 131-139.

Lisboa, C., Braga, L. L., \& Ebert, G. (2009). O fenômeno bullying ou vitimização entre pares na atualidade: Definições, formas de manifestação e possibilidades de intervenção. Contextos Clínicos, 2(1), 59-71.

Lopes, A. A. N. (2005). Bullying - comportamento agressivo entre estudantes. Jornal de Pediatria, 81(05), 164-172.

Malta, D. C., Silva, M. A. I., Mello, F. C. M., Monteiro, R. A., Sardinha, L. M. V., Crespo, C., Carvalho, M. G. O., Silva, M. M. A., \& Porto, D. L. (2010). Bullying nas escolas brasileiras: Resultados da Pesquisa Nacional de Saúde do Escolar (PeNSE), 2009. Ciência \& Saúde Coletiva, 15(2), 3065-3076.

McWilliam, N. (2010). A school peer mediation program as a context for exploring therapeutic jurisprudence (TJ): Can a peer mediation program inform the law? International Journal of Law and Psychiatry, 33(5-6), 293-305.

Merrell, K. W., Gueldner, B. A., Ross, S. W., \& Isava, D. M. (2008). How effective are school bullying intervention programs? A meta-analysis of intervention research. School Psychology Quarterly, 23(1), 26-42.

Neme, C. M. B., Mello, L. C., Gazzola, R. A., \& Justi, M. M. (2008). Fenômeno bullying: Análise de pesquisas em psicologia publicadas no período de 2000 a 2006. Revista Cibersaude.

Olweus, D. (1993). Bullying at school: What we know and what we can do. London: Lackwell.

Paludo, S. S., \& Koller, S. H. (2007). Psicologia Positiva: Uma nova abordagem para antigas questões. Paidéia, 17(36), 9-20.

Perkins, H. W., Craig, D. W., \& Perkins, J. M. (2011). Using social norms to reduce bullying: A research intervention among adolescents in five middle schools. Group Processes \& Intergroup Relations, 14(5), 703-722.

Petersen, C. S., \& Wainer, R. (2011). Terapias cognitivocomportamentais para crianças e adolescentes: Ciência e arte. Porto Alegre: Artmed.

Rigby, K. (1997). What children tell us about bullying in schools. Children Australia, 22(2), 28-34

Rolim, M. (2008). Bullying: O pesadelo da escola, um estudo de caso e notas sobre o que fazer. Dissertação de Mestrado, Programa de Pós-Graduação em Sociologia, Instituto de Sociologia, Universidade Federal do Rio Grande do Sul, Porto Alegre.

Salmivalli, C., Lagerspetz, K. M. J., Björkqvist, K., Österman, K., \& Kaukiainen, A. (1996). Bullying as group process: Participant roles and their relations to social status within the group. Aggressive Behaviour, 22, $1-15$.

Salmivalli, C., Kärnä, A., \& Poskiparta, E. (2011). Counteracting bullying in Finland: The KiVa program and its effects on different forms of being bullied. International Journal of Behavioral Development, 35(5), 405-411.

Schultz, N. C. W., Duque, D. F., Silva, C. M., Souza, C. D. Assini, L. C., \& Carneiro, M. G. M. (2012). A compreensão sistêmica do bullying. Psicologia em Estudo, 17(2), 247-254.

Seligman, M. E. P., \& Csikszentmihalyi, M. (2000). Positive psychology: An introduction. American Psychologist, 55, 5-14.

Triviños, A. N. S. (1987). Introdução à pesquisa em ciências sociais: A pesquisa qualitativa em educação. São Paulo: Atlas.

Tsang, S. K. M., Hui, E. K. P., \& Law, B. C. M (2011). Bystander position taking in school bullying: The role of positive identity, self-efficacy, and self-determination. The Scientific World Journal, 11, 2278-2286.

Ttofi, M. M., \& Farrington, D. P. (2011). Effectiveness of school-based programs to reduce bullying: A systematic and meta-analytic review. Journal of Experimental Criminology, 7, 27-56.

Twemlow, S. T., Biggs, B. K., Nelson, T. D., Vernberg., E. M., Fonagy, P., \& Twemlow, S. W. (2008). Effects of participation in a martial arts-based antibullying program in elementary schools. Psychology in the Schools, 45(10), 947-959.

Waasdorp, T. E., Bradshaw, C. P., \& Leaf, P. J. (2012). The impact of schoolwide positive behavioral interventions and supports on bullying and peer rejection. Archives of Pediatrics and Adolescent Medicine, 166(2), 149-56.

Williford, A., Boulton, A., Noland, B., Little, T. D., Kärnä, A., \& Salmivalli, C. (2012). Effects of the KiVa antibullying program on adolescents' depression, anxiety, and perception of peers. Journal of Abnormal Child Psychology, 40(2), 289-300.

Whitney, I., \& Smith, P.K. (1993). A survey of the nature and extent of bullying in junior/middle and secondary schools. Educational Research, 35, 3-25.

Submissão: $13 / 01 / 2014$
Primeira decisão editorial: $14 / 04 / 2016$
Aceite: $03 / 06 / 2016$

\section{Notas:}

1 Mestrado em Psicologia Clínica pela UNISINOS (2013) e Professora de Psicologia na Universidade FEEVALE. Contato: email: julianapureza@yahoo.com.br; Endereço: Av. Ipiranga, 6681- Partenon - Porto Alegre/RS - CEP: 90619-900, Prédio $11,9^{\circ}$ andar, 939

2 Doutorado (2009) em Psicologia pela Universidade Federal do Rio Grande do Sul. Atualmente é psicóloga clínica e professora do programa de pós-graduação em psicologia da Universidade do Vale do Rio dos Sinos (UNISINOS).

3 Doutorado pela Universidade Federal do Rio Grande do Sul, com estágio no exterior na Universidade do Minho em Portugal(2005). É bolsista Produtividade Nível 2/CNPq. Atualmente é Professora do Programa de Pós-Graduação em Psicologia e da Faculdade de Psicologia da Pontifícia Universidade Católica do Rio Grande do Sul, 